\title{
Vitamin Status as a Determinant of Serum Homocysteine Concentration in Type 2 Diabetic Retinopathy
}

\author{
Pandelis Fotiou, ${ }^{1}$ Athanasios Raptis, ${ }^{2}$ George Apergis, ${ }^{3}$ George Dimitriadis, ${ }^{2}$ \\ Ioannis Vergados, ${ }^{4}$ and Panagiotis Theodossiadis ${ }^{1}$ \\ ${ }^{1}$ 2nd Department of Ophthalmology, "Attikon" University Hospital, Athens University Medical School, 1 Rimini Street, \\ 12462 Athens, Greece \\ ${ }^{2}$ 2nd Department of Internal Medicine-Propaedeutic, Research Institute and Diabetes Center, "Attikon" University Hospital, \\ Athens University Medical School, 1 Rimini Street, 12462 Athens, Greece \\ ${ }^{3}$ Department of Molecular Diagnosis, "Hippokration" General Hospital, 114 Vasilissis Sofias Avenue, 11527 Athens, Greece \\ ${ }^{4}$ Athens Eye Hospital, 45 Vouliagmenis Avenue, 16675 Athens, Greece \\ Correspondence should be addressed to Pandelis Fotiou; panfotiou@hotmail.com
}

Received 21 May 2014; Accepted 24 May 2014; Published 10 June 2014

Academic Editor: Nikolaos Papanas

Copyright (c) 2014 Pandelis Fotiou et al. This is an open access article distributed under the Creative Commons Attribution License, which permits unrestricted use, distribution, and reproduction in any medium, provided the original work is properly cited.

We investigated the association of serum homocysteine levels and vitamin status with type 2 diabetic retinopathy. This study included 65 patients with and 75 patients without diabetic retinopathy. Patients with diabetic retinopathy had significantly higher serum homocysteine levels $(P<0.001)$, higher prevalence of hyperhomocysteinemia $(P<0.001)$, lower serum folic acid $(P<0.001)$, and vitamin $\mathrm{B}_{12}(P=0.014)$ levels than those without diabetic retinopathy. Regression analysis revealed that homocysteine was an independent risk factor for diabetic retinopathy and there was a threshold in its serum level $(13.7 \mu \mathrm{mol} / \mathrm{L})$, above which the risk of diabetic retinopathy greatly increases $(\mathrm{OR}=1.66, P=0.001)$. Folic acid was associated with decreased odds for diabetic retinopathy $(\mathrm{OR}=0.73, P<0.001)$. There was a threshold in serum vitamin $\mathrm{B}_{12}$ level $(248.4 \mathrm{pg} / \mathrm{mL})$, below which serum homocysteine concentration significantly increases with decreasing serum vitamin $\mathrm{B}_{12}(P=0.003)$. Our findings suggest that hyperhomocysteinemia is an independent risk factor for the development and progression of diabetic retinopathy. Decreased serum levels of folic acid and vitamin $\mathrm{B}_{12}$, through raising serum homocysteine concentrations, may also affect the diabetic retinopathy risk.

\section{Introduction}

Progressive disorders of the large and small vessels are common and serious complications of diabetes mellitus (DM). Studies over the last two decades have shown that hyperhomocysteinemia is associated with several macrovascular diseases, such as coronary artery disease, cerebrovascular disease, peripheral arterial disease, and deep-vein thrombosis [1-3].

Relatively few studies have investigated the association between hyperhomocysteinemia and diabetic retinopathy (DR) with yet inconsistent results. In addition, the vitamin status and its contribution to the development of DR have not been examined. In our study, we aimed to investigate whether there is an association between serum levels of homocysteine and those of folic acid and B-vitamins with DR in patients with type 2 diabetes mellitus (T2DM).

\section{Materials and Methods}

2.1. Subjects. The study group included 140 patients with T2DM, 69 men and 71 women, aged from 41 to 83 years (mean age $64.4 \pm 9.9$ years), all of Greek descent. All patients were recruited from the Ophthalmology Outpatient Clinics as well as the Diabetes Center of the "Attikon" University Hospital (Medical School of Athens University) in Athens, Greece. Diabetes was diagnosed according to the guidelines of the American Diabetes Association [4]. 
A detailed medical history was obtained from all study participants. The study included patients with known age of diagnosis of diabetes $>40$ years. We excluded patients with one or more of the following conditions:

(1) patients with a history of vascular disease (myocardial infarct or angina, stroke, peripheral arterial disease, and deep-venous thrombosis), renal, hepatic, chronic gastroenterologic, thyroid or blood disease, dementia, and neoplasm, since all these conditions are known to affect homocysteine blood concentrations;

(2) patients receiving vitamin supplementation or medications known to affect serum homocysteine concentrations, such as theophylline, statins, fibrates, levodopa, protons pump inhibitors, anticonvulsives, and contraceptives, and patients consuming good amounts of alcohol and heavy smokers;

(3) patients with uncontrolled arterial hypertension, defined as a systolic blood pressure $\geq 140 \mathrm{mmHg}$, and/or a diastolic blood pressure $\geq 90 \mathrm{mmHg}$;

(4) patients with uncertain diabetes duration.

The study was in accordance with the declaration of Helsinki and was approved by the Local Ethics Committee. Written informed consent was obtained from all subjects.

\subsection{Methods}

2.2.1. Ophthalmologic Examination. All patients underwent a complete ophthalmologic examination. Retinopathy was assessed by dilated ophthalmoscopy, fundus photography, and fluorescein angiography, when was indicated. Retinopathy was defined as the presence of any of such characteristic lesions: microaneurysms, hemorrhages, cotton wool spots, intraretinal microvascular abnormalities, hard exudates, venous beading, new vessels, laser scars, or a history of vitrectomy. Grading of the retinal findings was assessed according to the system used by the Early Treatment Diabetic Retinopathy Study Research Group [5]. Patients with diabetes were classified into two groups according to the findings of the "worst eye": 65 with DR (46.4\%) and 75 without DR (53.6\%). Of the 65 patients with DR, 36 (55.4\%) had nonproliferative diabetic retinopathy (NPDR) and 29 (44.6\%) had proliferative diabetic retinopathy (PDR).

2.2.2. Biochemical Analyses. Overnight fasting blood samples were taken from all subjects and were centrifuged within 1 hour following collection. The samples for homocysteine estimation were immediately chilled on ice and then were centrifuged and the serum was stored at $-20^{\circ} \mathrm{C}$. Serum glucose concentration was measured with a colorimetric assay on an automated analyzer MODULAR P800 (Roche Diagnostics $\mathrm{GmbH}$, Mannheim, Germany) and glycosylated hemoglobin $\left(\mathrm{HbA}_{1 \mathrm{C}}\right)$ with an immunoturbidimetric assay on an analyzer INTEGRA 400plus (Roche Diagnostics GmbH, Mannheim, Germany). Serum creatinine concentration was measured with the colorimetric assay (Jaffé) on the automated analyzer MODULAR P800. Folic acid and vitamin $B_{12}$ levels were estimated by electrochemiluminescence immunoassay on the analyzer MODULAR ANALYTICS E170 (Roche Diagnostics $\mathrm{GmbH}$, Mannheim, Germany) and those for vitamins $\mathrm{B}_{2}$ and $\mathrm{B}_{6}$ by high performance liquid chromatography (HPLC) with fluorescence detection (Chromsystem Diagnostics $\mathrm{GmbH}$, München, Germany). Estimation of serum homocysteine concentration was carried out by fluorescence polarization immunoassay (AXSYM SYSTEM, Abbott Diagnostics, Mannheim, Germany). Estimation of body weight is made by using the body mass index $(\mathrm{BMI})=\mathrm{kg} /$ height $(\mathrm{m})^{2}$.

2.3. Statistical Analysis. Differences between patients with and without DR in continuous variables were tested using $t$-test for two independent samples and, when normality assumption was violated, using the Mann-Whitney test. For categorical variables, chi square or Fisher's exact test was applied. Association of homocysteine with folic acid and vitamin $B_{12}$ was investigated using Spearman correlation coefficient, while when $\mathrm{HbA}_{1 \mathrm{C}}$ and diabetes duration groups were considered, one-way ANOVA or Kruskal-Wallis test (with Bonferroni correction when needed) was used. Differences by gender and type of retinopathy were tested by $t$-test for two independent samples or the Mann-Whitney test. The association of homocysteine with possible prognostic variables was also tested in multivariable regression models. This was a two-stage procedure. In the first stage, using generalized additive models (GAMs), the shape of the association with continuous variables was explored. When a threshold was identified, this was estimated using appropriate methodology. In the final regression model, those variables identified in the first step were included in the model using two linear parts, one below and one above the threshold (piecewise linear). Finally, multivariable logistic regression models were used to identify prognostic variables for DR (binary variable, yes/no) in the same way that was described above for homocysteine.

\section{Results}

All our results are presented in median (interquartile range) values. The demographic and laboratory data of the study population are shown in Table 1 . The age of patients with DR was statistically higher than that of patients without DR (68.0 (60.0-75.0) versus 61.0 (55.0-67.0), resp., $P=0.001$ ), as was also their positive family history of diabetes $(86.2 \%$ versus 54.7\% resp., $P<0.001$ ), and their BMI (29.2 (27.9-31.2 versus 27.8 (26.1-30.2), resp., $P=0.004)$. Patients with DR had a significantly longer diabetes duration (15.0 (10.0-22.0) versus 6.0 (3.0-9.0), $P<0.001$ ), higher serum glucose (154.0 (126.0188.0) versus 119.0 (103.0-136.0), $P<0.001), \mathrm{HbA}_{1 \mathrm{C}}$ (7.4 (6.6$8.9)$ versus $6.7(6.0-7.6), P<0.001)$, and serum creatinine level $(0.9(0.8-1.1)$ versus $0.9(0.7-1.0), P=0.024)$, but in both groups creatinine levels were well within the normal range. A significantly higher serum homocysteine level was observed in patients with DR than in those without DR (16.3 (14.7-19.8) versus 11.1 (9.5-13.6), resp., $P<0.001)$, and hyperhomocysteinemia (serum homocysteine $>15 \mu \mathrm{mol} / \mathrm{L}$ ) was noted in $70.8 \%$ of individuals with DR compared to $14.7 \%$ without DR $(P<0.001)$. As for the serum vitamins, patients 
TABLE 1: Demographic and laboratory characteristics of patients with type 2 diabetes mellitus.

\begin{tabular}{|c|c|c|c|}
\hline Characteristics & $\mathrm{DR}-(N=75)$ & $\mathrm{DR}+(N=65)$ & $P$ value \\
\hline Age (years) & $61.0(55.0-67.0)$ & $68.0(60.0-75.0)$ & 0.001 \\
\hline Positive family history (\%) & $54.7(N=41)$ & $86.2(N=56)$ & $<0.001$ \\
\hline $\operatorname{BMI}\left(\mathrm{kg} / \mathrm{m}^{2}\right)$ & $27.8(26.1-30.2)$ & $29.2(27.9-31.2)$ & 0.004 \\
\hline Diabetes duration (years) & $6.0(3.0-9.0)$ & $15.0(10.0-22.0)$ & $<0.001$ \\
\hline Fasting serum glucose $(\mathrm{mg} / \mathrm{dL})$ & $119.0(103.0-136.0)$ & $154.0(126.0-188.0)$ & $<0.001$ \\
\hline $\mathrm{HbA}_{1 \mathrm{C}}(\%)$ & $6.7(6.0-7.6)$ & $7.4(6.6-8.9)$ & $<0.001$ \\
\hline Serum creatinine $(\mathrm{mg} / \mathrm{dL})$ & $0.9(0.7-1.0)$ & $0.9(0.8-1.1)$ & 0.024 \\
\hline Serum homocysteine $(\mu \mathrm{mol} / \mathrm{L})$ & $11.1(9.5-13.6)$ & $16.3(14.7-19.8)$ & $<0.001$ \\
\hline Hyperhomocysteinemia (\%) & $14.7(N=11)$ & $70.8(N=46)$ & $<0.001$ \\
\hline Serum folic acid (ng/mL) & $11.0(8.3-17.0)$ & $8.7(7.2-10.3)$ & $<0.001$ \\
\hline Serum vitamin $\mathrm{B}_{2}(\mu \mathrm{g} / \mathrm{L})$ & $10.8(7.8-13.2)$ & $9.1(7.0-12.7)$ & 0.179 \\
\hline Serum vitamin $\mathrm{B}_{6}(\mu \mathrm{g} / \mathrm{L})$ & $11.6(9.9-13.2)$ & $11.0(8.0-14.6)$ & 0.481 \\
\hline Serum vitamin $B_{12}(\mathrm{pg} / \mathrm{mL})$ & $361.5(255.6-471.2)$ & $288.8(209.5-416.5)$ & 0.014 \\
\hline Systolic blood pressure (mmHg) & $125.0(115.0-130.0)$ & $130.0(120.0-135.0)$ & 0.016 \\
\hline Diastolic blood Pressure $(\mathrm{mmHg})$ & $75.0(70.0-80.0)$ & $75.0(70.0-80.0)$ & 0.409 \\
\hline
\end{tabular}

Values are presented as median (interquartile range) or \% (number).

TABLE 2: Association of serum homocysteine levels with diabetes duration and glycosylated hemoglobin.

\begin{tabular}{|c|c|c|c|}
\hline \multirow[t]{2}{*}{ Parameters } & & \multicolumn{2}{|c|}{ Homocysteine $(\mu \mathrm{mol} / \mathrm{L})$} \\
\hline & $\leq 5(N=42)$ & $11.2(8.7-13.9)$ & \multirow{5}{*}{$P$ value ${ }^{*}$} \\
\hline \multirow{4}{*}{ Diabetes duration (years) } & $6-15(N=60)$ & $12.2(10.0-16.2)$ & \\
\hline & $\geq 16(N=38)$ & $16.9(14.8-18.9)$ & \\
\hline & Overall $(N=140)$ & $12.9(10.3-16.9)$ & \\
\hline & $<6.5(N=43)$ & $11.2(9.5-15.8)$ & \\
\hline \multirow[t]{4}{*}{$\mathrm{HbA}_{1 \mathrm{C}}(\%)$} & $6.5-7.4(N=43)$ & $12.3(10.6-18.4)$ & \multirow{4}{*}{$P$ value $^{* *}$} \\
\hline & $7.5-8.4(N=26)$ & $15.0(11.1-16.2)$ & \\
\hline & $\geq 8.5(N=28)$ & $15.1(11.3-17.4)$ & \\
\hline & Overall $(N=140)$ & $12.9(10.3-16.9)$ & \\
\hline
\end{tabular}

* $\leq 5$ years versus $\geq 16 P<0.001,6-15$ years versus $\geq 16 P=0.001$, overall $P<0.001$.

${ }^{* *} P=0.126$.

Values are presented as median (interquartile range).

with DR had, though in the normal range, significantly lower folic acid (8.7 (7.2-10.3) versus $11.0(8.3-17.0), P<0.001)$ and vitamin $B_{12}$ (288.8 (209.5-416.5) versus 361.5 (255.6-471.2), $P=0.014)$ levels, whereas there was no significant difference for vitamin $B_{2}(9.1$ (7.0-12.7) versus 10.8 (7.8-13.2), $P=0.179)$ and vitamin $\mathrm{B}_{6}(11.0(8.0-14.6)$ versus 11.6 (9.9-13.2), $P=$ 0.481 ) levels between DR and no DR groups, respectively. Systolic blood pressure was higher in patients with DR (130.0 (120.0-135.0) versus 125.0 (115.0-130.0), $P=0.016)$, but no significant difference in diastolic blood pressure was noted between the two groups (75.0 (70.0-80.0) versus 75.0 (70.0$80.0), P=0.409)$. However, in all our diabetic patients blood pressure was within the normal range.

In Table 2, the data on the association between serum homocysteine levels and two significant parameters are shown. Serum homocysteine levels of diabetic patients were significantly increased with increasing diabetes duration $(\leq 5$ versus $\geq 16$ years, homocysteine: $11.2(8.7-13.9)$ versus 16.9 (14.8-18.9), $P<0.001,6-15$ versus $\geq 16$ years, homocysteine:
$12.2(10.0-16.2)$ versus 16.9 (14.8-18.9), $P=0.001$, overall $P<$ $0.001)$. However, serum homocysteine levels did not change significantly with increments in $\mathrm{HbA}_{1 \mathrm{C}}$ levels $\left(\mathrm{HbA}_{1 \mathrm{C}}<6.5\right.$ homocysteine: 11.2 (9.5-15.8), 6.5-7.4: 12.3 (10.6-18.4), 7.5-8.4: 15.0 (11.1-16.2), $\geq 8.5$ : 15.1 (11.3-17.4), $P=0.126)$.

There was a statistically significant difference in serum homocysteine levels between the NPDR group compared to the PDR group (NPDR: 15.5 (11.8-17.4), PDR: 18.7 (16.5-22.0), $P=0.001)$.

A statistically significant negative linear relationship was found between serum homocysteine and folic acid levels (Spearman $r=-0.261, P=0.002$ ), as well as between serum homocysteine and vitamin $\mathrm{B}_{12}$ levels (Spearman $r=-0.219$, $P=0.009$ ).

Multiple linear regression analysis revealed a significant association between serum homocysteine level and age (Coef.: 0.099, 95\% CI: 0.02, 0.18, $P=0.018$ ), diabetes duration (Coef.: 0.106, 95\% CI: 0.02, 0.20, $P=0.020$ ), and an inverse relationship with folic acid (Coef.: $-0.220,95 \%$ 
TABLE 3: Multiple linear regression analysis of the association of serum homocysteine with independent variables.

\begin{tabular}{|c|c|c|c|}
\hline & Coef. & $95 \% \mathrm{CI}$ & $P$ value \\
\hline Age (years) & 0.099 & $0.02,0.18$ & 0.018 \\
\hline Sex (male) & 0.859 & $-0.56,2.28$ & 0.233 \\
\hline BMI $\left(\mathrm{kg} / \mathrm{m}^{2}\right)$ & 0.149 & $-0.06,0.36$ & 0.166 \\
\hline Fasting serum glucose $(\mathrm{mg} / \mathrm{dL})$ & 0.005 & $-0.01,0.02$ & 0.523 \\
\hline Diabetes duration (years) & 0.106 & $0.02,0.20$ & 0.020 \\
\hline Folic acid $(\mathrm{ng} / \mathrm{mL})$ & -0.220 & $-0.37,-0.07$ & 0.005 \\
\hline Vitamin $\mathrm{B}_{12}^{\dagger}(\mathrm{pg} / \mathrm{mL})$ & -0.027 & $-0.04,-0.01$ & 0.003 \\
\hline Vitamin $\mathrm{B}_{12}{ }^{\ddagger}(\mathrm{pg} / \mathrm{mL})$ & -0.002 & $-0.01,0.00$ & 0.409 \\
\hline
\end{tabular}

${ }^{\dagger}$ below threshold.

‡above threshold.

TABLE 4: Multiple logistic regression analysis of the association of diabetic retinopathy with prognostic variables.

\begin{tabular}{lccc}
\hline & OR & $95 \%$ CI & $P$ value \\
\hline Age (years) & 1.03 & $0.97-1.09$ & 0.332 \\
Diabetes duration (years) & 1.18 & $1.08-1.28$ & $<0.001$ \\
$\mathrm{HbA}_{1 \mathrm{C}}(\%)$ & 2.30 & $1.49-3.54$ & $<0.001$ \\
Homocysteine $^{\dagger}(\mu \mathrm{mol} / \mathrm{L})$ & 1.66 & $1.24-2.23$ & 0.001 \\
Homocysteine $^{\ddagger}(\mu \mathrm{mol} / \mathrm{L})$ & 0.83 & $0.61-1.12$ & 0.219 \\
Folic acid $(\mathrm{ng} / \mathrm{mL})$ & 0.73 & $0.62-0.86$ & $<0.001$ \\
\hline
\end{tabular}

$\dagger^{\dagger}$ above threshold.

${ }^{\ddagger}$ below threshold.

CI: $-0.37,-0.07, P=0.005)$ and vitamin $B_{12}$ (Coef.: -0.027 , 95\% CI: $-0.04,-0.01, P=0.003)$. No associations with sex (Coef.: 0.859, 95\% CI: $-0.56,2.28, P=0.233$ ), BMI (Coef.: 0.149, 95\% CI: $-0.06,0.36, P=0.166$ ), and glucose (Coef.: 0.005, 95\% CI: $-0.01,0.02, P=0.523$ ) were observed (Table 3). There is a threshold in the exposure-response association of serum homocysteine with vitamin $\mathrm{B}_{12}$, below which changes in serum vitamin $B_{12}$ levels significantly affect serum homocysteine concentration (turning point: 248.4, standard error: 43.8) (Figure 1).

Multiple logistic regression analysis also showed that variables that independently affect $\mathrm{DR}$ risk were diabetes duration (OR: 1.18, 95\% CI: 1.08, 1.28, $P<0.001$ ), $\mathrm{HbA}_{1 \mathrm{C}}$ (OR: 2.30, 95\% CI: 1.49, 3.54, $P<0.001$ ), and homocysteine concentrations (OR: 1.66, 95\% CI: 1.24, 2.23, $P=0.001$ ). Folic acid conferred a protective effect on the DR risk (OR: $0.73,95 \%$ CI: $0.62,0.86, P<0.001$ ) (Table 4). There is a threshold in the association of homocysteine with DR (turning point: 13.7, standard error: 1.4). For every increase of serum homocysteine by $1 \mu \mathrm{mol} / \mathrm{L}$ above the threshold, there is an increased risk of about $66 \%$ for the development of DR (Figure 2).

\section{Discussion}

The results of our study revealed significantly higher concentration of serum homocysteine as well as a higher prevalence of hyperhomocysteinemia (serum homocysteine $>15 \mu \mathrm{mol} / \mathrm{L}$ ) in patients with T2DR compared to those without DR and hyperhomocysteinemia as an independent risk factor for DR. Our results also revealed a threshold

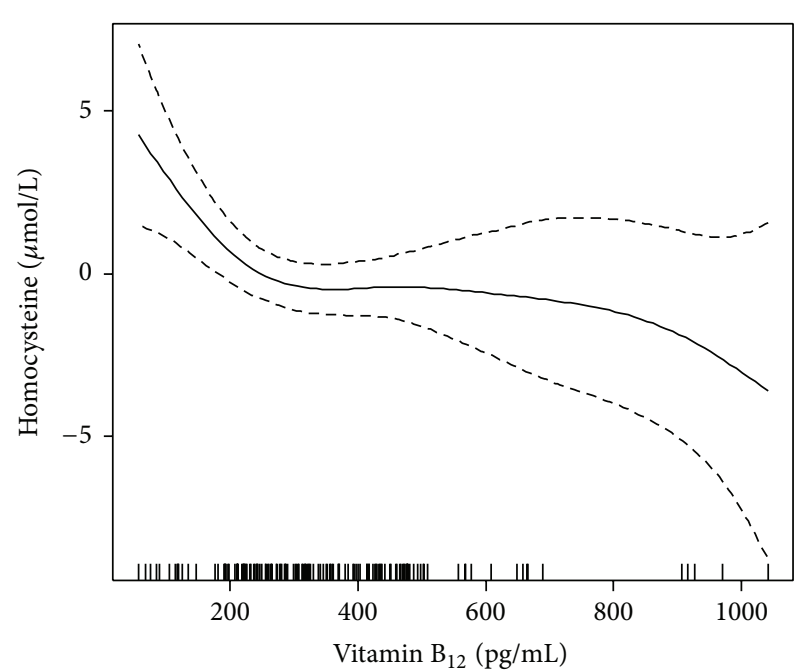

FIGURE 1: Shape of association between serum homocysteine and $\mathrm{B}_{12}$.

in the association of serum homocysteine level with DR risk of around $14.0 \mu \mathrm{mol} / \mathrm{L}$, above which a $1 \mu \mathrm{mol} / \mathrm{L}$ increase in serum homocysteine concentration was an independent predictor of increased DR risk of $66 \%$. A higher serum homocysteine level in patients with PDR than in those with NPDR was also found; a result that may suggest that homocysteine is related not only to the development but also to the progression of DR. There are a number of studies that have evaluated the association between hyperhomocysteinemia and type $2 \mathrm{DR}$ but have so far yielded inconsistent 


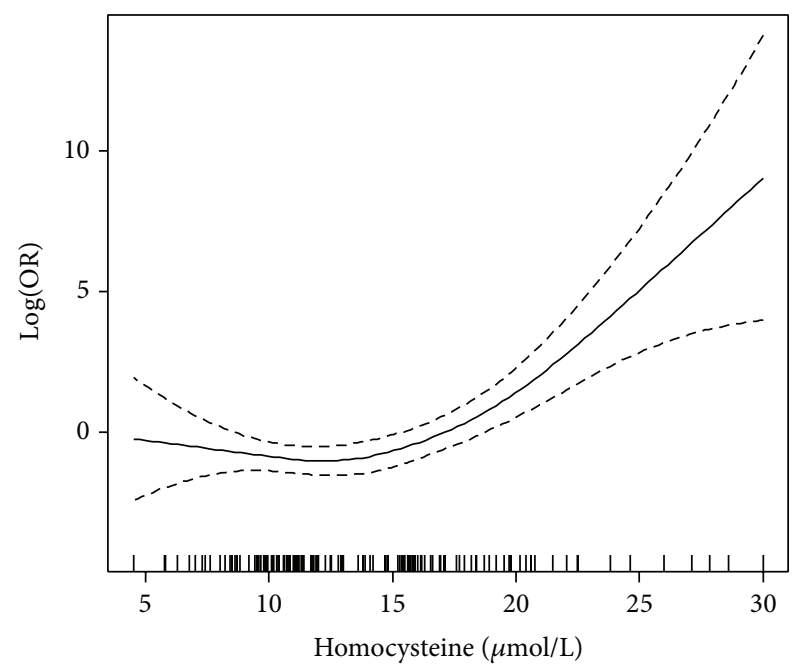

FIGURE 2: Shape of association between DR risk and serum homocysteine.

results [6-9]. The differences in the findings among studies are most likely related to differences in type of diabetes or to inadequately controlling for confounding factors, such as nephropathy and hypertension, but also lifestyle (e.g., vitamin intake) and genetic factors. In addition, the cutoff level for hyperhomocysteinemia differed substantially among studies (from 11.0 to $16.0 \mu \mathrm{mol} / \mathrm{L}$ ).

The exact pathogenesis of DR is multifactorial and remains largely unclear but may involve (1) endothelial dysfunction and (2) low-grade chronic inflammation of the retinal capillaries. Hyperhomocysteinemia promotes these two pathophysiologic mechanisms [10]. It has long been recognized that oxidative stress is associated with the progression of diabetes and its complications. The adverse effects of hyperhomocysteinemia on the endothelium may be triggered by increased oxidative stress in the diabetic vasculature. Hyperhomocysteinemia increases NADPH oxidase activity [11], promotes uncoupling of endothelial nitric oxide synthase [12], and inhibits the function of intracellular antioxidant enzymes, such as glutathione peroxidase and superoxide dismutase [13]. Moreover, autooxidation of excess homocysteine may directly lead to additional ROS production [14]. Accumulating ROS reacts with nitric oxide (NO) to form peroxynitrite radicals, leading to decreased NO bioavailability and activity and subsequent endothelial dysfunction.

ROS activate inflammatory mediators and cytokines (interleukins, tumor necrosis factor- $\alpha$ ). In addition, upregulation of ROS leads to activation of proinflammatory mediators, such as the nuclear factor- $\kappa \mathrm{B}$, in retinal capillary cells, which further increasing the expression of cytokines [15]. These inflammatory events result in increased endothelial cell expression of adhesion molecules, which contribute to leucocyte accumulation and attachment to the retinal capillaries (leucostasis) [16]. Leucostasis, which is believed to be an early event in DR, may lead to the blood-retinal barrier breakdown and eventually to chronic leucocyte mediated cell damage and death [17].
With regard to the risk factors for DR, regression analysis of our data confirmed that poor glycemic control and longer diabetes duration are independent risk factors for the development and progression of DR $[18,19]$. In our study, the group of patients without DR succeeded in achieving the target mean $\mathrm{HbA}_{1 \mathrm{C}}$ level of $7.0 \%$ or less [4] $\left(\mathrm{HbA}_{1 \mathrm{C}} 6.9 \pm 1.1 \%\right)$ but this was not the case with the group of patients with DR $\left(\mathrm{HbA}_{1 \mathrm{C}} 7.8 \pm 1.5 \%\right)$. However, it is true that perfect glycemic control is very difficult to attain for most diabetic patients and glucose control has the tendency to deteriorate with time. A significant finding in our study was the association of increasing serum homocysteine concentration with longer diabetes duration. Thus, we may infer that the known harmful effect of DM duration on the development of retinopathy and its progression is not only related to the long-standing hyperglycemia but also to the increased homocysteine concentrations with time and its subsequent adverse effects on retinal capillaries. On the contrary, we did not find an association between homocysteine concentrations and increasing $\mathrm{HbA}_{1 \mathrm{C}}$ levels. This may be due either to an actual lack of such an association between the two factors or to the inconsistency of hyperglycemia during the progression of DM. Besides, evidence from both healthy and diabetic twins indicates that $\mathrm{HbA}_{1 \mathrm{C}}$ levels are determined for a significant part by genetic factors. This provides evidence that $\mathrm{HbA}_{1 \mathrm{C}}$ is in part determined by factors other than glycemic control and may account for the variation in $\mathrm{HbA}_{1 \mathrm{C}}$ levels among people $[20,21]$.

Methionine and homocysteine metabolism depends upon adequate stores of folic acid and vitamins $B_{2}, B_{6}, B_{12}$, which act as cofactors or substrates in the metabolism and are important nutritional determinants of serum homocysteine. A number of studies have shown an inverse association between blood folic acid and homocysteine concentration $[22,23]$. Such an inverse relationship between folic acid and homocysteine levels was also observed in our study. In regard with vitamin $B_{12}$, the reason for the limited number of studies with this vitamin is that its deficiency is rather unusual, especially in Western countries, due to the fact that the vitamin is present in food of animal origin. A recent study from India, where the prevalence of micronutrient deficiency is reported to be high, mentions a high prevalence of vitamin $\mathrm{B}_{12}$ deficiency in the diabetic population (54-67\%). Mean vitamin $B_{12}$ level in the $\mathrm{DR}$ group was below the normal range and higher homocysteine levels were significantly associated with lower vitamin $B_{12}$ levels [24]. We also detected such an inverse association between vitamin $B_{12}$ and homocysteine levels. Our study illustrates lower levels of folic acid and vitamin $B_{12}$ in patients with $D R$. It is noteworthy that low, but not necessarily deficient, levels of these two vitamins contributed to the development of hyperhomocysteinemia in our study. Regression analysis also showed that folic acid confers a protective effect on the development of DR. Another significant observation in our study was the finding of a threshold of about $250 \mathrm{pg} / \mathrm{mL}$ of serum vitamin $B_{12}$, below which there is a sharp rise in serum homocysteine with decreasing vitamin $B_{12}$ levels.

Vitamin $B_{6}$ deficiency is extremely unusual, because this vitamin is present in a wide range of food of animal and plant 
origin. In our study, we found no cases of biochemical vitamin $\mathrm{B}_{6}$ deficiency. One study has reported an association between low vitamin $\mathrm{B}_{6}$ and vascular disease that was unrelated to hyperhomocysteinemia, but it was explained by a relationship between low vitamin $\mathrm{B}_{6}$ status and chronic inflammation, which is known to promote DR [25]. The results of our study show that vitamin $\mathrm{B}_{6}$ levels were well within the normal range in patients with diabetes irrespective of the presence of retinopathy. Vitamin $B_{2}$ acts in the form of flavin adenine dinucleotide (FAD), which is a substrate for the enzyme methylene tetrahydrofolate reductase (MTHFR). This enzyme is the most important genetic determinant of blood homocysteine in the general population. In our study material, no cases of biochemical vitamin $B_{2}$ deficiency were observed, neither do we know any such studies, and the levels of this vitamin were also well within the normal range in all of our diabetic patients. Increased dietary folic acid and vitamin $\mathrm{B}_{2}$ requirements have been observed mainly in persons with the genetic variant of the enzyme MTHFR, C677T [26]. Studies confirm that vitamin $B_{2}$ is an independent determinant of homocysteine in TT homozygotes of the C677T polymorphism [27].

In conclusion, among the many factors, some of them are yet unidentified, that affect the development and progression of DR, hyperhomocysteinemia seems to be associated with $\mathrm{DR}$, at least as a biomarker. Longer diabetes duration and lower folic acid and vitamin $B_{12}$ status appear to be important determinants for hyperhomocysteinemia in DR patients. Monitoring serum homocysteine concentration, as well as folate and vitamin $\mathrm{B}_{12}$ status in T2DM patients, could be used as an indicator for assessing microvascular risk in DM. Treatment of existing hyperhomocysteinemia with folic acid and vitamin $\mathrm{B}_{12}$ may be useful in reducing the risk of microvascular complications in T2DM.

\section{Conflict of Interests}

The authors declare that there is no conflict of interests regarding the publication of this paper.

\section{Acknowledgments}

The authors declare that no financial support has been received for this paper. The authors are grateful to $\mathrm{Mr}$. Antonis Analitis (Statistician, Department of Hygiene, Epidemiology and Medical Statistics, Medical School, University of Athens, Athens, Greece) for his excellent work on the statistics of this paper.

\section{References}

[1] L. L. Humphrey, R. Fu, K. Rogers, M. Freeman, and M. Helfand, "Homocysteine level and coronary heart disease incidence: a systematic review and meta-analysis," Mayo Clinic Proceedings, vol. 83, no. 11, pp. 1203-1212, 2008.

[2] Homocysteine Studies Collaboration, "Homocysteine and risk of ischemic heart disease and stroke: a meta-analysis," The Journal of the American Medical Association, vol. 288, no. 16, pp. 2015-2022, 2002.
[3] N. Khandanpour, Y. K. Loke, F. J. Meyer, B. Jennings, and M. P. Armon, "Homocysteine and peripheral arterial disease: systematic review and meta-analysis," European Journal of Vascular and Endovascular Surgery, vol. 38, no. 3, pp. 316-322, 2009.

[4] American Diabetes Association, "Diagnosis and classification of diabetes mellitus," Diabetes Care, vol. 35, supplement 1, pp. 564-571, 2012.

[5] Early Treatment Diabetic Retinopathy Study Research Group, "Grading diabetic retinopathy from stereoscopic color fundus photographs - an extension of the modified Airlie House classification. ETDRS report number 10," Ophthalmology, vol. 98, supplement 5, pp. 786-806, 1991.

[6] N. A. Abdella, O. A. Mojiminiyi, A. O. Akanji, and M. A. Moussa, "Associations of plasma homocysteine concentration in subjects with type 2 diabetes mellitus," Acta Diabetologica, vol. 39, no. 4, pp. 183-190, 2002.

[7] H. C. Looker, A. Fagot-Campagna, E. W. Gunter et al., "Homocysteine as a risk factor for nephropathy and retinopathy in type 2 diabetes," Diabetologia, vol. 46, no. 6, pp. 766-772, 2003.

[8] E. K. Hoogeveen, P. J. Kostense, P. E. D. Eysink et al., "Hyperhomocysteinemia is associated with the presence of retinopathy in type 2 diabetes mellitus: the Hoorn study," Archives of Internal Medicine, vol. 160, no. 19, pp. 2984-2990, 2000.

[9] L. Brazionis, K. Rowley Sr., C. Itsiopoulos, C. A. Harper, and K. O'Dea, "Homocysteine and diabetic retinopathy," Diabetes Care, vol. 31, no. 1, pp. 50-56, 2008.

[10] M. V. van Hecke, J. M. Dekker, G. Nijpels et al., "Inflammation and endothelial dysfunction are associated with retinopathy: the Hoorn study," Diabetologia, vol. 48, no. 7, pp. 1300-1306, 2005.

[11] Z. Ungvari, A. Csiszar, J. G. Edwards et al., "Increased superoxide production in coronary arteries in hyperhomocysteinemia: role of tumor necrosis factor- $\alpha$, NAD $(\mathrm{P}) \mathrm{H}$ oxidase, and inducible nitric oxide synthase," Arteriosclerosis, Thrombosis, and Vascular Biology, vol. 23, no. 3, pp. 418-424, 2003.

[12] L. Jin, R. B. Caldwell, T. Li-Masters, and R. W. Caldwell, "Homocysteine induces endothelial dysfunction via inhibition of arginine transport," Journal of Physiology and Pharmacology, vol. 58, no. 2, pp. 191-206, 2007.

[13] E. Lubos, J. Loscalzo, and D. E. Handy, "Homocysteine and glutathione peroxidase-1," Antioxidants and Redox Signaling, vol. 9, no. 11, pp. 1923-1940, 2007.

[14] A. Andersson, A. Lindgren, and B. Hultberg, "Effect of thiol oxidation and thiol export from erythrocytes on determination of redox status of homocysteine and other thiols in plasma from healthy subjects and patients with cerebral infarction," Clinical Chemistry, vol. 41, no. 3, pp. 361-366, 1995.

[15] L. Papatheodorou and N. Weiss, "Vascular oxidant stress and inflammation in hyperhomocysteinemia," Antioxidants and Redox Signaling, vol. 9, no. 11, pp. 1941-1958, 2007.

[16] A. P. Adamis, "Is diabetic retinopathy an inflammatory disease?" The British Journal of Ophthalmology, vol. 86, no. 4, pp. 363-365, 2002.

[17] R. Chibber, B. M. Ben-Mahmud, S. Chibber, and E. M. Kohner, "Leukocytes in diabetic retinopathy," Current Diabetes Reviews, vol. 3, no. 1, pp. 3-14, 2007.

[18] Diabetes Control and Complications Trial Research Group, "Progression of retinopathy with intensive versus conventional treatment in the Diabetes Control and Complications Trial," Ophthalmology, vol. 102, pp. 647-661, 1995. 
[19] R. Klein, B. E. K. Klein, S. E. Moss, and K. J. Cruickshanks, "Relationship of hyperglycemia to the long-term incidence and progression of diabetic retinopathy," Archives of Internal Medicine, vol. 154, no. 19, pp. 2169-2178, 1994.

[20] H. Snieder, P. A. Sawtell, L. Ross, J. Walker, T. D. Spector, and R. D. G. Leslie, "HbAlc levels are genetically determined even in type 1 diabetes: evidence from healthy and diabetic twins," Diabetes, vol. 50, no. 12, pp. 2858-2863, 2001.

[21] R. M. Cohen, H. Snieder, C. J. Lindsell et al., "Evidence for independent heritability of the glycation gap (glycosylation gap) fraction of HbAlc in nondiabetic twins," Diabetes Care, vol. 29, no. 8, pp. 1739-1743, 2006.

[22] J. Selhub, P. F. Jacques, P. W. F. Wilson, D. Rush, and I. H. Rosenberg, "Vitamin status and intake as primary determinants of homocysteinemia in an elderly population," The Journal of the American Medical Association, vol. 270, no. 22, pp. 2693-2698, 1993.

[23] O. Nygård, H. Refsum, P. M. Ueland, and S. E. Vollset, "Major lifestyle determinants of plasma total homocysteine distribution: the Hordaland Homocysteine study," The American Journal of Clinical Nutrition, vol. 67, no. 2, pp. 263-270, 1998.

[24] A. Satyanarayana, N. Balakrishna, S. Pitla et al., "Status of Bvitamins and homocysteine in diabetic retinopathy: association with Vitamin- $B_{12}$ deficiency and hyperhomocysteinemia," PLoS ONE, vol. 6, no. 11, Article ID e26747, 2011.

[25] S. Friso, P. F. Jacques, P. W. F. Wilson, I. H. Rosenberg, and J. Selhub, "Low circulating vitamin $\mathrm{B}_{6}$ is associated with elevation of the inflammation marker C-reactive protein independently of plasma homocysteine levels," Circulation, vol. 103, no. 23, pp. 2788-2791, 2001.

[26] P. F. Jacques, A. G. Bostom, R. R. Williams et al., "Relation between folate status, a common mutation in methylenetetrahydrofolate reductase, and plasma homocysteine concentrations," Circulation, vol. 93, no. 1, pp. 7-9, 1996.

[27] H. McNulty, K. Pentieva, L. Hoey, and M. Ward, "Homocysteine, B-vitamins and CVD," Proceedings of the Nutrition Society, vol. 67, no. 2, pp. 232-237, 2008. 


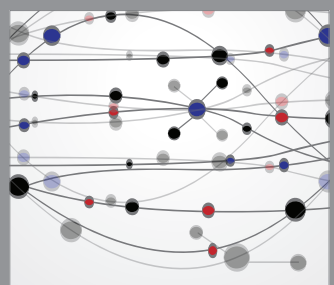

The Scientific World Journal
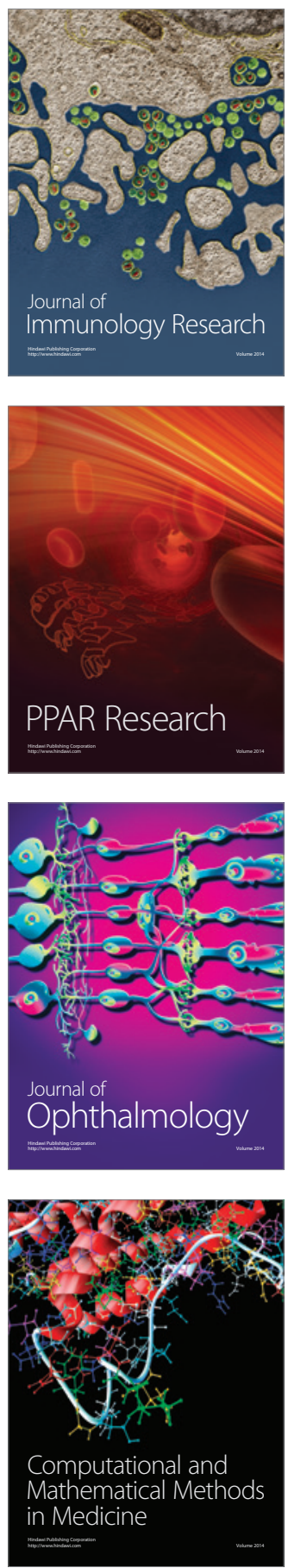

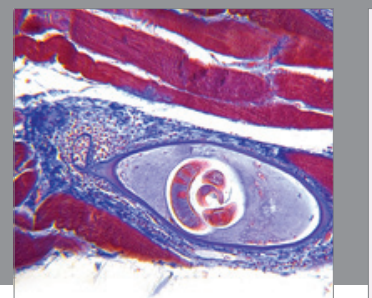

Gastroenterology

Research and Practice
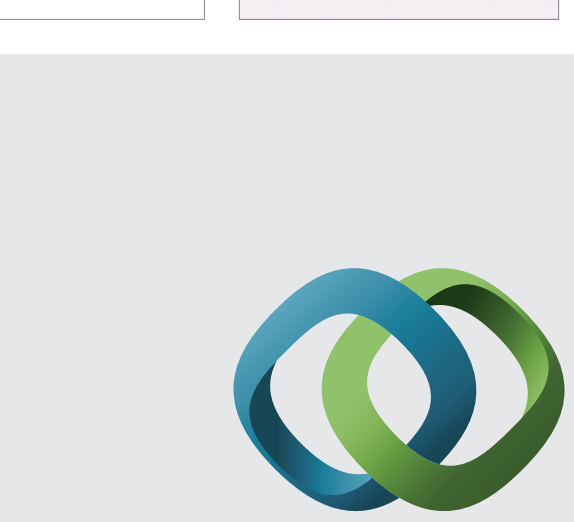

\section{Hindawi}

Submit your manuscripts at

http://www.hindawi.com
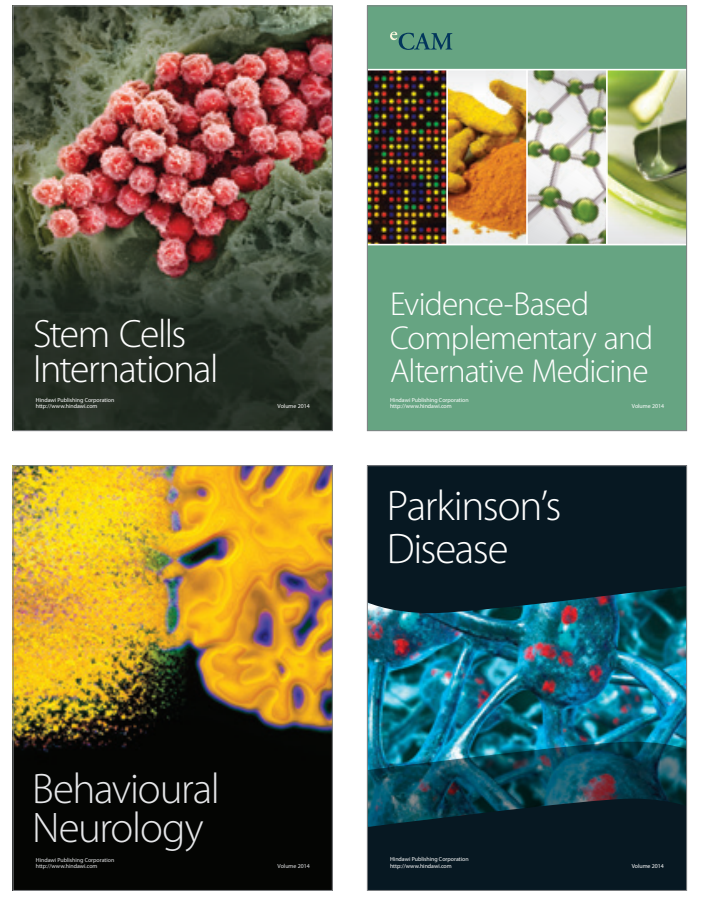
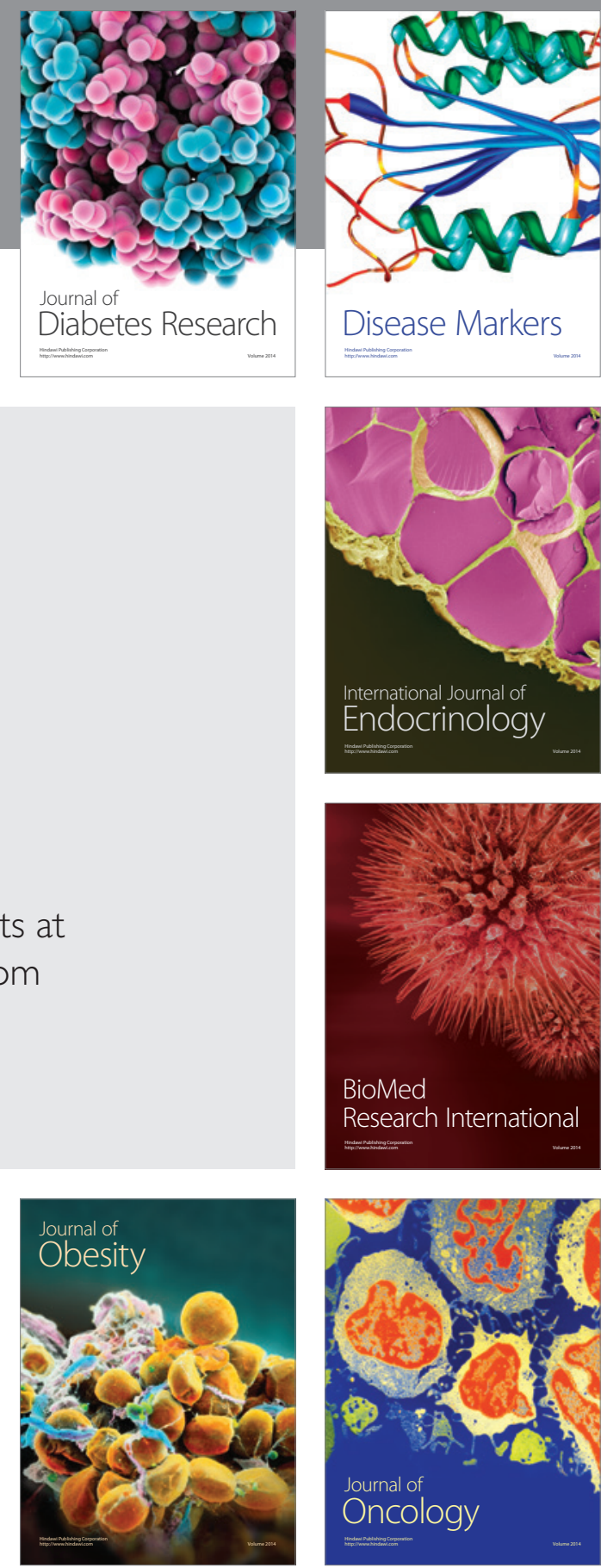

Disease Markers
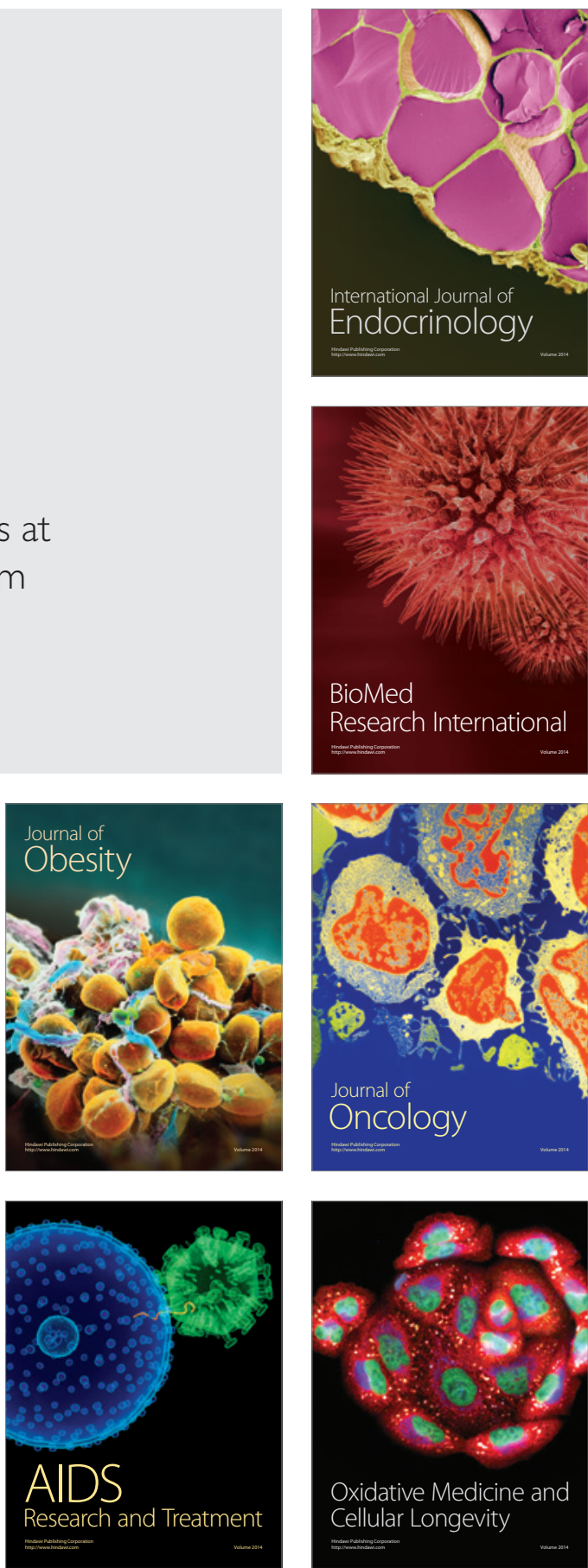